\title{
Clinical and cranial computed tomography scan findings in adults following road traffic accidents in Kampala, Uganda.
}

\author{
Geoffrey Erem ${ }^{1}$, Samuel Bugeza ${ }^{1}$, Elsie Kiguli Malwadde ${ }^{1,2}$
}

1. Makerere University, College of Health Sciences, Department of Radiology.

2. African Centre for Global Health and Social Transformation (ACHEST)

\begin{abstract}
Background: Globally, road traffic accidents are a major cause of death and disability. The developing countries bear a disproportionately large share of the RTAs which account for about $85 \%$ of the deaths. Most of these RTAs result in head injury, which globally, most scholars and medical practitioners consider a significant economic, social and medical problem. In Mulago National referral hospital, RTA is the leading cause of surgical admission.

Objective: To describe the cranial computed tomography (CT) scan findings in adults following RTA in Mulago hospital.

Methods: Using CT, detailed analysis of 178 adult patients with head injury following RTA was performed. Data was analyzed using SPSS version 16 and presented in tables and graphs. Data recorded included socio-demographic characteristics, clinical and $\mathrm{CT}$ variables.

Results: Seventy seven percent of the respondents were between 18- 39 years. 52.6\% of patients had open head injury. Headache was the most common clinical variable followed by dizziness and aphasia. The most common CT characteristic was extra cerebral haemorrhage followed by brain oedema and raised Intra-cranial pressure (ICP). Intra-cerebral haemorrhage was commonest in the frontal lobe followed by parietal lobe.

Conclusion: Public health interventions like advocacy and education of the population on safe and responsible road usage should be emphasized to reduce on RTAs.

Keywords: Computed tomography scan, road traffic accidents, head injury, Uganda.

DOI: https://dx.doi.org/10.4314/ahs.v17i1.15

Cite as: Erem G, Bugeza S, Malwadde EK. Clinical and cranial computed tomography scan findings in adults following road traffic accidents in Kampala, Uganda. Afri Health Sci. 2017;17(1): 116-121. https://dx.doi.org/10.4314/abs.v17i1.15
\end{abstract}

\section{Introduction}

Globally, road traffic accidents (RTAs) are a major cause of death and disability with a disproportionate number accruing in developing countries ${ }^{1}$. Road traffic accidents account for $2.1 \%$ of global mortality ${ }^{2}$. The developing countries bear a large share of the burden and account for about $85 \%$ of the deaths as a result of RTAs ${ }^{2}$. The problem is increasing at a fast rate in developing countries due to rapid motorization and other factors ${ }^{3}$. Public policy responses to this epidemic have been muted nationally and internationally ${ }^{4}$. Road traffic accidents are the leading cause of surgical admissions at Mulago hospital. This is
Corresponding author:
Geoffrey Erem,
Makerere University College of Health
Sciences, School of Medicine, Radiology Department,
Mulago Hospital, Kampala, Uganda.
Phone number: +256791368974.
Email: dreremgeoffrey@gmail.com

mainly because of reckless taxi operators on poorly designed roads 5 . Head injury is a significant economic, social and medical problem all over the world. Injury and deaths due to road traffic accidents are a major public health problem in the developing countries. Road traffic accidents are the most frequent cause of head injury with highest risk among the young age group. ${ }^{5}$ The study by Galukande et al showed that $35.5 \%$ of patients present to Mulago hospital with head injury following RTA in Kampala ${ }^{5}$.

About 4000 CT scan examinations were done in a year at Mulago hospital in 2011, 44\% of these were due to RTA with clinical evidence of head injury. The high burden of imaging in Uganda means that hospitals dedicate a significant amount of time and resources to trauma management ${ }^{6}$. There are no recent studies detailing the patterns of head injury following RTA in Uganda. Previous studies were done over ten years ago, could the pattern of head injury have changed over time? There is need to bridge this knowledge gap. Therefore, the purpose of 
this study was to describe the cranial CT scan findings following RTA.

\section{Material and methods \\ Study setting}

This was a descriptive, cross sectional study conducted in Mulago national referral hospital in Uganda with a bed capacity of 1500 and bed occupancy rate of 180 to $200 \%$. Emergency brain CT scan service is available on a 24 hour basis.

\section{Sample size and sampling procedures}

Adult patients recruited in this study were admitted through accident and emergency department to the emergency ward via Radiology department for brain CT scan examination. The sample size was calculated using open info computer software with the following assumptions: Population size of patients presenting to Mulago over 4 months with head injury $587(\mathrm{~N})$, a study by Kobusingye et al in 1999 showed that road traffic injuries account for $35.1 \%+/-5$ of all trauma in Kampala (p) with confidence limits as $\%$ of $100($ absolute $+/-\%)(\mathrm{d})=5 \%$ and a design effect $(\mathrm{DEFF})=1$. Using the equation Sample size $\mathrm{n}=[\mathrm{DEFF} * \mathrm{~Np}(1-\mathrm{p})] /\left[\left(\mathrm{d} 2 / \mathrm{Z} 21-\alpha / 2 *(\mathrm{~N}-1)+\mathrm{p}^{*}(1-\mathrm{p})\right], \mathrm{a}\right.$ sample size (n) of 178 patients was calculated and subsquently recruited in the study.

\section{Equipment}

A Philips Tomoscan MX16, Host model, August 2010 CT machine in the department of Radiology was used to perform cranial CT scan examinations. Patients were stabilized in the emergency room before CT scan examination. No special patient preparation was required for emergency brain CT scans examinations. Non-enhanced helical axial brain CT scans were done from the skull base to the vertex. The images were annotated and a hard copy printed in bone and soft tissue windows. A discussed report of the examination and copies of the images were immediately availed to the Neurosurgeon on duty. Discussion points included the presence or absence of lesions, size of lesion, density of lesion, site of lesion, raised ICP which was evaluated by presence of effacement or obliteration of the basal cisterns especially the ambient and quadrigerminal cisterns. These were all captured in the data collection tool.

\section{Recruitment procedure for participants}

Informed consent was sought from the patients who met the inclusion criterion. All patients were scanned irrespective of their ability to pay for the examination or not. All the study patients had their history taken and examined by the admitting doctor in accident and emergency department. Brain CT scan examination was performed by the CT Technician on duty, and interpretations done by the principal investigator and discussed with the co-authors. The information was captured in the data forms. Data was collected using pre-tested questionnaires.

\section{Statistical methods}

A statistician consultation was done during the planning of the study to ensure orderly collection, recording, editing, storage, retrieval and rigorous data analysis. Data sets were coded, cleaned and entered into a computer using EPIDATA software version 3.1 and exported to SPSS computer software version 16 for analysis. Frequency distributions were computed for different clinical variables such as dizziness, headache, aphasia, convulsions, open or closed head injury and hemiparesis as well as CT variables such as extra-cerebral haemorrhage, intra-cerebral haemorrhage, midline shift, brain oedema, raised ICP and skull fractures.

\section{Ethical considerations}

Permission to carryout the study was sought from Ethics and Research Committee of Makerere University College of Health Sciences. Aware that almost all biomedical research are conducted for the purpose of systematically collecting and analyzing data from which generalisable conclusions may be drawn that may aid in improving the care of currently unknown beneficiaries in the future. We selected research participants in a fairly and equitable manner without personal bias. Lastly, we ensured that research participants' privacy was protected, we gave them opportunities to withdraw from the study should they decide otherwise.

\section{Results}

\section{Socio-demographic characteristics of the patients}

A total of 178 patients with head injury following road traffic accidents were recruited in the study. Majority of the patients were below 40 years, mean age was 32.6 years (range $18-80$ years) with a median of 49 years and mode of $20-29$ years.

Of the 178 patients who had head injury following RTA, males accounted for $144(80.9 \%)$ translating into a ratio of 4.2: 1 (Figure 1). 


\section{Figure 1: Distribution of head injury by sex $(\mathrm{N}=178)$.}

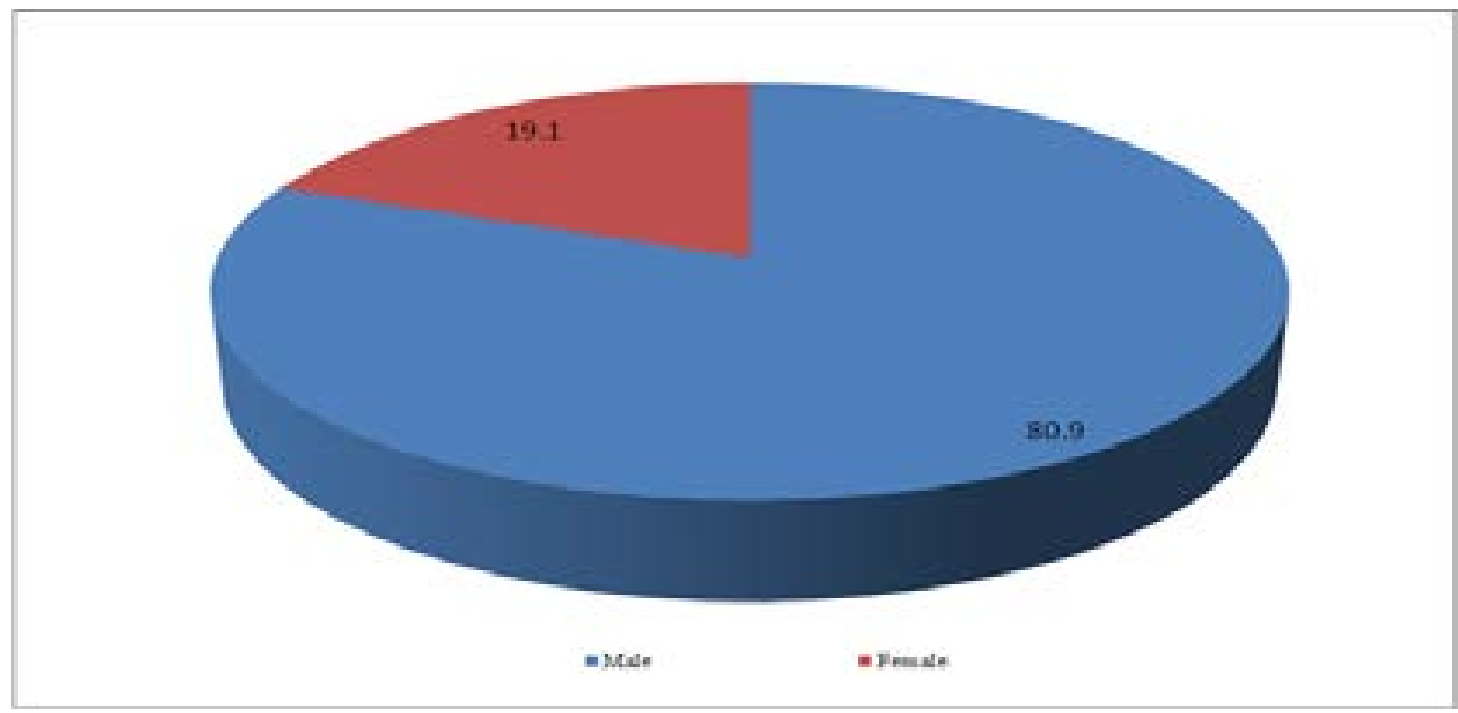

Boda boda (44\%) cyclists were the most involved in RTA, servants $(37 \%)$ and the least involved being the unemclosely followed by business men/women (39\%), public ployed (3\%) (Figure 2).

\section{Figure 2: Distribution of head injury by occupation $(\mathrm{N}=178)$.}

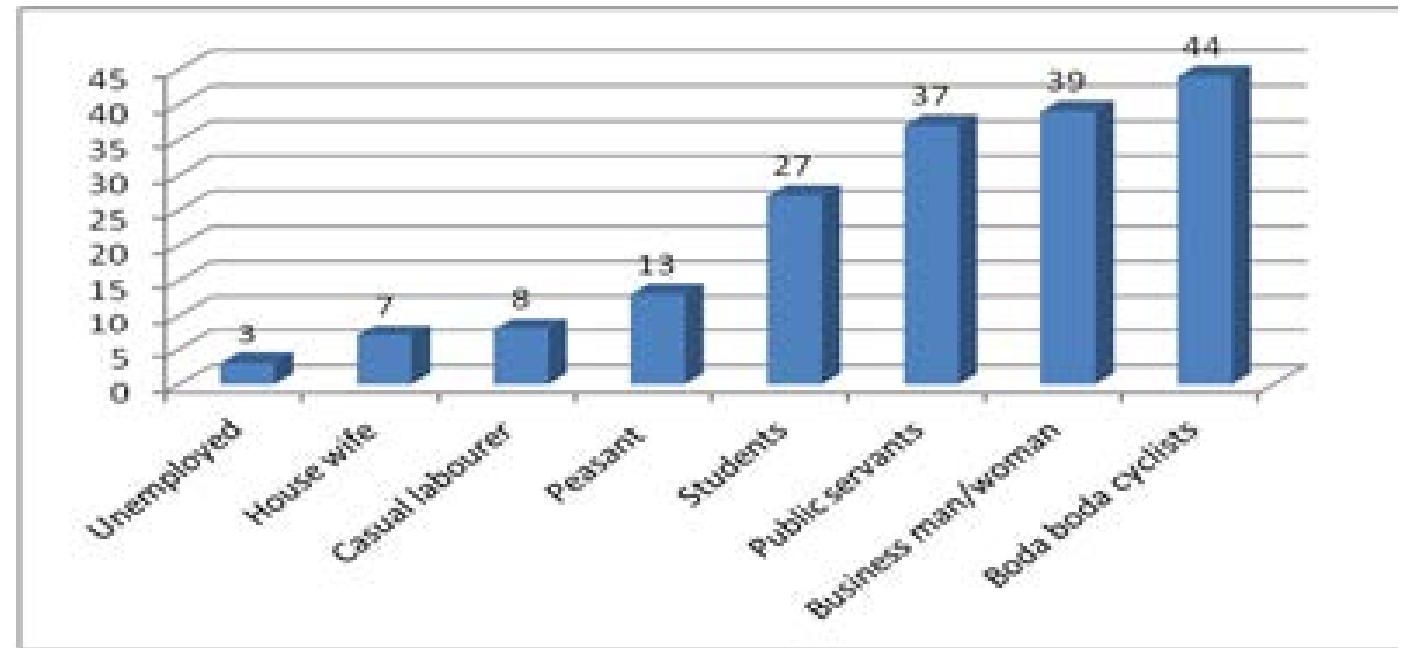

Clinical findings of the study participants.

About $53 \%$ of patients had open head injury, the rest had closed head injury. Headache was the most common clinical variable $(73 \%)$ followed by dizziness $(58 \%)$ and aphasia $(42 \%)$. About $14 \%$ of the patients had convul- sions, while $76 \%$ of them had generalized convulsions. Of those who had altered mentation, $58 \%$ had a GCS of 13-15. This was followed by about 30\% with a GCS of 9-12. Only $12 \%$ of the patients had a GCS of less than 8 (Table 1). 


\section{Table 1: Socio-demographic characteristics of the patients studies $(\mathrm{N}=178)$}

Characteristics
Age
$<20$
$20-29$
$30-39$
$40-49$
250
Marital status
Married
Single
Widower/widowed
Divorced
Distance from Hospital
O-5 Km
$6-10$ Km
$>10$ Km
Cause of accident
Bicycle
Boda boda
Motor vehicle
Unknown
Passenger detail
Carpassenger
Boda boda cyclist
Driver
Motor cycle passenger
Pedestrian
Influence of alcohol
Yes
No

$\begin{array}{ll}\text { Number of patients }(\mathbf{n}) & \text { Percentage (\%) } \\ 21 & 12.1 \\ 73 & 41.3 \\ 42 & 23.6 \\ 21 & 11.5 \\ 21 & 11.5 \\ 104 & 58.4 \\ 63 & 35.4 \\ 8 & 4.5 \\ 3 & 1.7 \\ 12 & 6.7 \\ 49 & 27.5 \\ 117 & 65.7 \\ 2 & \\ 95 & 1.1 \\ 75 & 53.4 \\ 6 & 42.1 \\ 19 & 3.4 \\ 68 & 10.7 \\ 12 & 38.2 \\ 29 & 6.7 \\ 50 & 16.3 \\ 27 & 28.1 \\ 151 & 15.2 \\ & 84.8\end{array}$

\section{Cranial computed tomography findings}

The most common CT characteristic was extra-cerebral haemorrhage (44.1\%). This was closely followed by brain oedema $(38.8 \%)$, raised ICP $(36.7 \%)$, normal brain $(35.4 \%)$ and skull fractures $(34.5 \%)$. Intra-cerebral hae- morrhage was most common in the frontal lobe $(28.7 \%)$ followed by the parietal lobe $(25.6 \%)$. The occipital lobe $(2.2 \%)$ and the cerebellum $(0.6 \%)$ were the least involved parts of the brain. Thirty eight $(21.6 \%)$ of patients had a midline shift of more than $3 \mathrm{~mm}$, seven $(4 \%)$ of whom were more than $10 \mathrm{~mm}$ (Table 2).

\section{Table 2: Clinical findings of the study participants}

Clinical variables
Dizziness
Headache
Aphasia
Convulsions
Focal
Generalized
Clinical diagnosis
Open head injury
Closed head injury
Hemiparesis
Left
Right
Bilateral
Pupils
Normal
Dilated
Unilateral
Bilateral
Constricted
Unilateral
Bilateral
GCS
$13-15$
$9-12$
$\leq 8$

Frequency (n)

Percentage (\%)

104
129
74
25
6
19

92
83
12
6
4
2

127
5
2
3
6
1
5
102
53
21




\section{Discussion}

Analysis of data indicated that the most common age group involved in head injuries following RTAs was 20 - 29 years. A look at the Uganda's employment sector shows that the youth form the bulk of the employment sector engaging in both formal and informal economic activities.

The youth are also very mobile in the course of their work making them prone to RTAs. This is in keeping with previous studies conducted in 2009 by Galukande and Naddumba ${ }^{7,8}$.

Furthermore, the results indicate a male preponderance of $4.2: 1$, this is also similar to previous studies done in 1998 and 2004 in Uganda ${ }^{7,8,10}$. The typical cyclists who use bicycles and motorcycles in Kampala and surrounding areas were young adult males.

Most of the patients with head injury following RTAs cannot be managed in upcountry hospitals, so they are referred for specialised Neuro-surgical care at the National referral hospital. The Neuro-surgeons are also only based at the national hospital since they are very few.

The study showed that boda boda cyclists are the common group of people involved in RTAs, Motorcycles commonly referred to as boda bodas contributed to the highest burden of head injuries in this paper closely followed by motor vehicles. This is because of recklessness, indiscipline and lack of respect for other road users by most motorist all over the country ${ }^{7,8}$. There is also rapid motorization of Kampala, the roads are poorly planned, narrow with no designated passages for pedestrians. The pedestrians were the most commonly injured during road traffic accidents in this study ${ }^{4}$.

Our study also showed that most patients had mild head injury at presentation with only a few having severe head injury. The most common clinical variable at presentation included headache, dizziness, ENT bleeding and aphasia. Contrary to our findings where the most common clinical diagnosis was open head injury, earlier studies in Kampala reported closed head injury as the most common clinical diagnosis at presentation ${ }^{8}$.

Our findings further show that the most common CT characteristic was extra-cerebral haemorrhage, closely followed by brain oedema, raised ICP, normal brain and skull fractures respectively. These findings differ from previous studies where brain oedema and sub-dural haematoma were the most common CT findings ${ }^{10}$. This dif- ference could probably be due to the change in motorisation pattern since the last study was done about 10 years ago in Uganda.

Intra-cerebral haemorrhage was commonest in the frontal lobe followed by the parietal lobe. The occipital lobe and the cerebellum were the least injured areas of the brain. This distribution of the intra-parenchymal haemorrhage is consistent with studies done locally and internationally ${ }^{8-10}$. This could partly be explained by the mechanism of impact in boda boda cyclists who were the most common group involved in RTAs, they usually fall forwards or sideways. Besides, most boda boda cyclists or their passengers rarely wear protective head helmets as seen in the study by Galukande ${ }^{6}$. This leaves the skull unprotected in the event of road traffic accidents.

\section{Conclusion and recommendations}

Intra-cerebral haemorrhage, brain oedema, raised ICP, skull fractures and normal brain were diagnoses following CT scan examinations. The pattern of cranial CT characteristics has changed over time in Kampala, Uganda.

Public health interventions like advocacy and education of the population on safe and responsible road usage should be emphasized to reduce on RTAs.

\section{Acknowledgement}

We would like to acknowledge the staff of Radiology and the Radiographers who scanned the patients, Mr. Ogwal Ronald who was the research assistant in this study that emanated to the production of this paper. At the same time, we extend our special gratitude to Professor Jacob Godfrey Agea for his effort and guidance in reviewing this article.

\section{Conflict of interest}

There is no conflict of interest to declare.

\section{References}

1. Howe J, Davis A (2002). Boda Boda - Uganda's Rural and Urban low capacity Transport Services, Independent Tropical Consultants UK .

2. M Peden, L Sminkey. World Health Organization dedicates World Health Day to road safety. Inj Prev 2004; 10:67.

3. Jacobs G, Aaron-Thomas A, Astrop A. Estimating global road traffic fatalities. London: Transport Research Laboratory, 2000. (TRL report 445) 
4. Krug E, ed. Injury: a leading cause of global burden of disease. Geneva: WHO, 1999. www.who.int/violence-injury-prevention/index.html.

5. Galukande M. Boda-boda Injuries a Health Problem and a Burden of Disease in Uganda: A Tertiary Hospital survey. East and Central African Journal of Surgery July/August 2009; 14(2):33-37.

6. Galukande M. Boda-boda Injuries a Health Problem and a Burden of Disease in Uganda: a Tertiary Hospital survey. East and Central African Journal of Surgery July/August 2009; 14(2):33-37.

7. Naddumba EK. A Cross sectional retrospective study of Boda Boda injuries at Mulago Hospital in Kampala, Uganda. East and Central African Journal of Surgery. 2004; Vol 9 No1: 44-47.

8. E. Kiguli-Malwadde, M G Kawooya , D Iga ,Matovu The value of $\mathrm{CT}$ in the management of head trauma patients at Mulago Hospital. East and Central African Journal of Surgery. June 1998 Vol 4: Vol. 43-42

9. Sosin DM, Sniezek JE, Thurman DJ. Incidence of mild and moderate brain injury in the United States, 1991. Brain Inj 10: 47-54, 1996.

10. Kawooya M, Kiguli M et al. Assessing the diagnostic imaging needs for five selected hospitals in Uganda. $J$ of clinical imaging science vol 1: issue 3: July-Sept 2011. 